\title{
Jets and photons spectroscopy of Higgs-ALP interactions
}

\author{
Alexandre Alves, ${ }^{a}$ A.G. Dias $^{b}$ and D.D. Lopes ${ }^{b}$ \\ ${ }^{a}$ Departamento de Física, Universidade Federal de São Paulo, \\ Diadema, 09913-030, Brasil \\ ${ }^{b}$ Centro de Ciências Naturais e Humanas, Universidade Federal do ABC, \\ Santo André-SP, Brasil \\ E-mail: aalves@unifesp.br, alex.dias@ufabc.edu.br, \\ lopes.diego@ufabc.edu.br
}

ABSTRACT: Axion-like particles (ALPs) and Higgs bosons can interact in scalar sectors beyond the Standard Model, leading the Higgs boson to decay into pairs of gluons and photons through the ALP interaction and giving rise to resonances in the decay products of the process $h \rightarrow a a \rightarrow g g+\gamma \gamma$, resembling a spectral-line analysis. We explore this signature to constrain an ALP effective field theory formulation. Our analyses show that the forthcoming runs of the LHC will be capable of probing the ALP-Higgs interaction in the ALP mass range from 0.5 to $60 \mathrm{GeV}$ using an automatized search strategy that adapts to different ALP masses in inclusive jets plus photons final states. Such interaction can also be tested in mass regions where the two and four-photon search channels are currently ineffective.

Keywords: Jets, Phenomenological Models

ArXIV EPRINT: 2105.01095 


\section{Contents}

1 Introduction 1

2 Effective Lagrangian and decay widths 4

3 Collider constraints $\quad 5$

4 Simulation details and search analysis $\quad 6$

4.1 Selection criteria - Higgs-ALP spectroscopy 8

5 Scanning the parameter space $\quad 13$

6 Results 14

7 Conclusions and prospects $\quad 16$

\section{Introduction}

The scalar sector of the Standard Model (SM) is currently much less constrained than the gauge and matter sectors, turning it into a target to the forthcoming runs of the LHC and future colliders as well. Many aspects of the scalar potential remain to be elucidated, including the possibility of new interactions. One plausible possibility is that the Higgs boson, $h$, has a coupling to a light pseudoscalar, $a$, allowing the decay $h \rightarrow a a$. Such pseudoscalars may arise in beyond Standard Model (BSM) theories as pseudo NambuGoldstone bosons of spontaneously broken quasi-exact symmetries, potentially involved in long-sought solutions of problems left open by the SM. Well known examples are axions and axion-like particles ALPs (for reviews of axions and ALPs see, for example, [1-8]). Axions are the pseudo Nambu-Goldstone bosons of an anomalous global chiral $\mathrm{U}(1)_{P Q}$ symmetry (broken at the quantum level and so generating a mass for the axion), which is required to solve the strong CP problem through the Peccei-Quinn mechanism [9-12]. The axion mass and its couplings to gauge and matter fields are inversely proportional to the energy scale at which the $\mathrm{U}(1)_{P Q}$ symmetry is broken spontaneously. In the most common viable axion models, that scale is very high, leading to tiny masses and couplings which, although being targeted by several experiments and confronted with astrophysical data $[1,6,7]$, cannot produce direct observable effects in collisions at the LHC.

On the other hand, ALPs do not necessarily share a mass-coupling relation like the axion. Their masses and couplings to SM particles become independent parameters and, thus, more amenable for phenomenological studies; at the same time, it gets easier to avoid experimental constraints. The ALP mass can arise, at the classical level, from a small parameter realizing the explicit breaking of the global symmetry associated with the ALP. 
In the limit where this parameter vanishes, the ALP turns into a Nambu-Goldstone boson. We can assume that the approximate symmetry associated with the ALP is a global U(1) symmetry whose spontaneous breaking occurs at a high energy scale $f$, the ALP decay constant. Although this scale is expected to be above the electroweak scale, it may not severely suppress the ALP couplings to the SM particles, allowing the observation of ALP effects at the LHC. It is also natural for the ALP to have a mass $m_{a} \ll f$ due to the approximate character of the $\mathrm{U}(1)$ symmetry.

New physics is expected around $f$, but below this energy scale, we have an effective theory for the ALP with its couplings to the SM particles having the cutoff scale $\Lambda \sim f$ as a suppression factor. A whole range of the ALP mass versus coupling to the Higgs boson can be investigated through the hypothetical decay $h \rightarrow a a$ by studying possible ALP decay modes in photons and gluons. As we will show, the mass region $0.5 \leq m_{a} \leq 60 \mathrm{GeV}$ can be well explored using an automatized search strategy that adapts itself to different ALP masses in inclusive jets plus photons final states. It is worthwhile to mention that some models predict heavy axions that could also be observed at colliders [13-15].

Many studies have already addressed an augmented Higgs scalar sector with pseudoscalars in ultra-violet completed theories. Good examples are the two Higgs doublet models [16], with the pseudo-scalar mass arising from a softly broken global symmetry in the scalar potential, and the Next-to-Minimal Supersymmetric Standard Model [17, 18]. However, instead of a top-down approach, we might look firstly for effective interactions between the SM and the new scalar particle. We will then focus on generic ALPs using the effective theory approach, i.e., without assuming a particular model for them, looking for their masses and couplings to the Higgs boson, gluons, and photons that could give a potential signal at the LHC within a region of the parameter space not previously explored.

Considering that the $\mathrm{U}(1)$ symmetry is realized non-linearly as the shift symmetry $a \rightarrow a+\alpha$, with $\alpha$ constant, the lowest order interaction occurs through a dim- 6 operator of the form $\frac{1}{\Lambda^{2}}\left(\partial_{\mu} a\right)\left(\partial^{\mu} a\right) \phi^{\dagger} \phi$, where $\phi$ is the Higgs doublet. After the electroweak symmetry breaking, this operator induces the Higgs to ALPs decay if it is kinematically allowed. Effective operators of ALP-gluons and ALP-photons are generally expected to be generated at the one-loop level in ultra-violet completed theories, where couplings with new heavy charged and colored fermions $\psi$ occur through renormalizable operators like iy a $\bar{\psi} \gamma_{5} \psi$. In the case of ALPs, besides their probable role in the solutions to unanswered questions in particle physics, light axions/ALPs extend their scope to other observed anomalies if we consider, for example, couplings to photons and leptons that might explain the $4.2 \sigma$ discrepancy of the $(g-2)_{\mu}$ data [19], the excess in excited Beryllium decays [20], and the excess in the gamma rays flux from the center of the galaxy observed by Fermi-LAT [21, 22], for instance.

At the LHC, theoretical studies and experimental searches for the SM Higgs boson decaying to ALPs have involved final states with jets, leptons, $Z$ bosons, and photons in various ALP mass regions [19, 23-31]. If the ALP mass is below the lightest pion threshold, the decay to photons becomes the most relevant one unless the couplings to electrons and muons get strong. Above the pion threshold, however, the decay to gluons involves detecting rare signals in an overwhelming background of QCD jets. The preferred 
option thus remains to search for multiphoton signals but with rates that die out as the ALP decays to hadrons become dominant.

Assuming that couplings to gluons and photons are dominant, detecting the Higgs decays to ALP pairs, $h \rightarrow a a$, can be done in the following basic channels depending on the ALP mass, $m_{a}$ : (1) four photons, $a a \rightarrow \gamma \gamma \gamma \gamma$; (2) two photons plus two gluons, $a a \rightarrow g g \gamma \gamma$ (3) four gluons, $a a \rightarrow g g g g$ when the ALP is heavy. If the ALP is light enough, its decay yields get collimated in the lab frame and, in this case, they are reconstructed as a single photon/jet, giving rise to signals like (4) $a a \rightarrow \gamma \gamma$; (5) $a a \rightarrow j \gamma$; (6) $a a \rightarrow j j$. (Hereafter, $j$ denotes a gluon jet as we assume that the ALP couples predominantly to gauge bosons). However, due to the detector finite resolution, any signal of the form $n j+m \gamma$, with $0 \leq(n, m) \leq 4,2 \leq n+m \leq 4$, is expected. ${ }^{1}$

Whatever is the ALP mass, the decays to jets exclusively cannot be disentangled from QCD backgrounds leaving the multiphoton as the prime option. Indeed, if we do not consider ALP-fermion couplings as relevant, $h \rightarrow a a \rightarrow \gamma \gamma$ is the best channel to constrain the Higgs-ALP coupling if $m_{a} \lesssim 400 \mathrm{MeV}$, and $h \rightarrow a a \rightarrow \gamma \gamma \gamma \gamma$ is a good option for $m_{a}>$ few $\mathrm{GeV}$. However, the mass range from $400 \mathrm{MeV} \lesssim m_{a} \lesssim$ few $\mathrm{GeV}$, where the hadronic decays are turned on, remains not accessible for multiphoton and multijet states.

We show that this mass gap can be probed at the $13 \mathrm{TeV}$ LHC in the $p p \rightarrow h \rightarrow$ $a a \rightarrow j(j)+\gamma(\gamma)$ channel where the ALP decays get eventually collimated enough to form a single photon or jet hit in the detector. In the SM, such Higgs decays are either forbidden by color charge conservation or loop-suppressed, and observing such a signal would be striking evidence for Higgs-ALP interactions. Our inclusive signal considers at least one isolated jet and one isolated photon; that is, we accept events with one and two isolated photons plus any number of isolated jets, which leads us to tackle many background sources with jets and photons. To clear our signals from the backgrounds, we employ an automatized kinematic cut strategy which permits us to probe the effective Higgs-ALP interaction assuming various model scenarios in the mass range from $0.5 \mathrm{GeV}$, where the hadronic decays open, up to $60 \mathrm{GeV}$, the Higgs to ALP decay threshold. Our analysis is especially effective in the mass gap from 0.5 to $\sim 10 \mathrm{GeV}$ where two- and four-photons final state searches lose sensitivity. Our search strategy explores the Higgs and ALP resonances in photons and jets final states by identifying the various spectral lines in the invariant mass distributions to determine whether the Higgs might contain ALPs in its composition.

The $h \rightarrow a a \rightarrow g g \gamma \gamma$ channel has already been investigated in refs. [29, 33]. The ATLAS Collaboration constrains the Higgs production cross section times the branching ratio of $h \rightarrow a a \rightarrow g g \gamma \gamma$ in the $20-60 \mathrm{GeV}$ mass region. We take these limits into account in our analysis. In ref. [33], which appeared before the Higgs boson discovery, the ALP pair was assumed as being the dominant Higgs decay mode for Higgs masses from 80 to $160 \mathrm{GeV}$. Contrary to ours, those analyses consider just the fully resolved final state with two isolated jets and two isolated photons with a fixed cut strategy. As we will show, to explore the whole mass region from 0.5 to $60 \mathrm{GeV}$ with maximum sensitivity, it is necessary to focus on different resonances and adjust the selection requirements for each ALP mass.

\footnotetext{
${ }^{1}$ Even resolved photons can be clustered into single "photon-jets" so the number of resolved and unresolved photons is, to some extent, a matter of definition [32].
} 
The paper is organized as follows: in section 2, we present the effective interaction Lagrangian and the relevant decay widths of the Higgs and the ALP bosons; in section 3, we enumerate and discuss the collider constraints imposed on the search for the ALP signals at the LHC; sections 4 and 5 are devoted to details of simulations, analysis and scanning of the parameters of the model; we present our results in section 6 ; section 7 contains the conclusions.

\section{Effective Lagrangian and decay widths}

Let us consider the following effective interaction Lagrangian, with the SM fields in the symmetry basis,

$$
\mathscr{L}_{\mathrm{eff}}=\frac{k_{B B}}{\Lambda} a B^{\mu \nu} \tilde{B}_{\mu \nu}+\frac{k_{W W}}{\Lambda} a W_{i}^{\mu \nu} \tilde{W}_{\mu \nu}^{i}+\frac{k_{G G}}{\Lambda} a G_{a}^{\mu \nu} \tilde{G}_{\mu \nu}^{a}+\frac{C_{h a}^{\mathrm{eff}}}{\Lambda^{2}} \partial_{\mu} a \partial^{\mu} a \phi^{\dagger} \phi
$$

where $\phi$ is the Higgs doublet field, $W_{\mu \nu}, B_{\mu \nu}$, and $G_{\mu \nu}^{a}$ are the $\mathrm{SU}(2)_{L}, \mathrm{U}(1)_{Y}$ and $\mathrm{SU}(3)_{C}$ field strength tensors, respectively. The pseudoscalar ALP field is denoted by $a$. The couplings of the ALP with the weak gauge interaction eigenstates $B$, and $W^{i}, i=1,2,3$ are given by $k_{B B}$ and $k_{W W}$, respectively, while $k_{G G}$ is the coupling between ALPs and gluons. The dual field $\tilde{B}_{\mu \nu}$ is defined as $\frac{1}{2} \varepsilon_{\alpha \beta \mu \nu} B^{\alpha \beta}$, with the totally anti-symmetric tensor being such that $\varepsilon_{0123}=1$, and analogously to the other fields. Taking the unitary gauge $\phi=[0(h+v) / \sqrt{2}]^{T}$, where $h$ is the Higgs boson and $v=246 \mathrm{GeV}$, the last term in eq. (2.1), which respects a shift symmetry $a \rightarrow a+\alpha$ of the ALP field, gives rise to the ALP-Higgs interaction operator $v C_{h a}^{\text {eff }} / \Lambda^{2} \times\left(\partial_{\mu} a\right)\left(\partial^{\mu} a\right) h$. As we mentioned before, $\Lambda$ is the effective Lagrangian cutoff, directly related to the ALP decay constant.

In our analysis, the relevant partial decay widths $h \rightarrow a a, a \rightarrow \gamma \gamma$, and $a \rightarrow g g$ are given by

$$
\begin{aligned}
\Gamma(h \rightarrow a a) & =\frac{v^{2}\left|C_{h a}^{\mathrm{eff}}\right|^{2}}{32 \pi \Lambda^{4}} m_{h}^{3}\left(1-\frac{2 m_{a}^{2}}{m_{h}^{2}}\right)^{2} \sqrt{1-\frac{4 m_{a}^{2}}{m_{h}^{2}}} \\
\Gamma(a \rightarrow \gamma \gamma) & =\frac{\left(k_{B B} c_{W}^{2}+k_{W W} s_{W}^{2}\right)^{2}}{4 \pi \Lambda^{2}} m_{a}^{3}, \\
\Gamma(a \rightarrow g g) & =\frac{8 k_{G G}^{2}}{4 \pi \Lambda^{2}} m_{a}^{3},
\end{aligned}
$$

with the sine(cossine) of the electroweak mixing angle defined as $s_{W}\left(c_{W}\right) \equiv \sin \theta_{W}\left(\cos \theta_{W}\right)$, while the total width of the ALP is computed from

$$
\Gamma_{a}=\Gamma(a \rightarrow \gamma \gamma)+\Gamma(a \rightarrow g g),
$$

assuming that the ALP couplings to gauge bosons are dominant. The ALP mass range that we are interested in does not allow decays to $W$ and $Z$ bosons.

In principle, the model can be parametrized by 5 independent parameters: the ALP mass, $m_{a}$, and the four effective couplings $k_{B B} / \Lambda, k_{W W} / \Lambda, k_{G G} / \Lambda$, and $C_{a h}^{\text {eff }} / \Lambda^{2}$. The coupling of the ALP to photon, however, occurs only through the combination $k_{\gamma \gamma} / \Lambda \equiv$ $\left(k_{B B} c_{W}^{2}+k_{W W} s_{W}^{2}\right) / \Lambda$. 
Moreover, once we consider a minimum setup where ALPs decay only to gluons and photons, their branching ratios must add to unity. Hence, the signal can be conveniently parametrized by the ALP mass, $m_{a}$, the ALP to photons branching ratio, $B R(a \rightarrow \gamma \gamma)$, and the effective Higgs-ALP coupling, $C_{a h}^{\text {eff }} / \Lambda^{2}$. Next, we present the collider constraints taken into account in our analysis.

\section{Collider constraints}

The $h \rightarrow a a \rightarrow j(j)+\gamma(\gamma)$ channel can be probed at the LHC for those model parameters that lead to short-lived ALPs with masses above the hadronic threshold. We also must avoid parameters already excluded by previous searches in the Higgs channels and searches dedicated exclusively to ALP detection. Right below, we present the $95 \%$ confidence level (CL) constraints imposed on the parameter space points that can be probed at the LHC. We assume that the Higgs boson is produced in gluon-fusion with the SM rate.

1. ALP lifetime - the ALP must decay before reaching the electromagnetic calorimeter. The distance that the ALP produced in the decay of the Higgs boson travels from the interaction point before decaying is given approximately by

$$
l_{\text {decay }} \approx \frac{m_{h}}{m_{a} \Gamma_{a}} \times 10^{-16} \mathrm{~m},
$$

where the ALP total width is given by eq. (2.3).

Therefore, in order to have a decay inside the ATLAS or the CMS detector, $l_{\text {decay }} \sim 1$ meter, we require that

$$
\frac{m_{a}}{m_{h}} \Gamma_{a}>10^{-16} \mathrm{~m} .
$$

2. $h \rightarrow j j \gamma \gamma$ - the direct search in the channel of our interest from the ATLAS Collaboration [29]. In this work, limits from

$$
\frac{\sigma_{H}}{\sigma_{\mathrm{SM}}} \times B R(h \rightarrow a a \rightarrow g g \gamma \gamma)<B R_{95}^{j j \gamma \gamma}\left(m_{a}\right),
$$

in the $20-60 \mathrm{GeV}$ ALP mass region, were obtained. Here, $\sigma_{H}$ and $\sigma_{\mathrm{SM}}$ are the inclusive production cross section of $p p \rightarrow h$ in the BSM and the SM scenarios, respectively, and $B R_{95}^{j j \gamma \gamma}\left(m_{a}\right)$ are the ALP mass dependent limits on that ratio. These limits are not so strong as the next ones that we consider, but they are important once they target part of the search channel that we are studying.

3. $h \rightarrow \gamma \gamma \gamma \gamma$ - the ATLAS Collaboration has performed a search for $h \rightarrow a a \rightarrow \gamma \gamma \gamma \gamma$ in the mass range of 10 to $62.5 \mathrm{GeV}$ in ref. [28] resulting in the following limit

$$
B R(h \rightarrow a a) \times B R^{2}(a \rightarrow \gamma \gamma)<(3-4) \times 10^{-4} .
$$

4. The Higgs boson total width - the Higgs boson total width is constrained, from onshell and off-shell Higgs production in the four-lepton final state [34], to be: $\Gamma_{h}=$ $3.2_{-2.2}^{+2.8} \mathrm{MeV}$, while the SM theoretical value is $\Gamma_{h}=4.1_{-4.0}^{+5.0} \mathrm{MeV}$. Thus, if we suppose 
that the difference between the upper bound of $\Gamma_{h}^{e x p}$ and the SM value of $4.07 \mathrm{MeV}$ is due only to the contribution of a single new channel, then

$$
B R(h \rightarrow a a) \leq \frac{6.0-4.1}{6.0}=32.2 \% .
$$

We will assume that dimension-5 operators such as $\frac{C_{z h}^{\text {eff }}}{\Lambda}\left(\partial_{\mu} a\right) Z^{\mu} h$ do not play a role in our analysis; that is, we assume $C_{a h}^{\mathrm{eff}} \gg C_{z h}^{\mathrm{eff}}$. Our analysis is complementary to those carried out for dimension-5 operators from refs. [30, 35].

5. Photonic decays of the $Z$ boson - the ALP masses considered in this work permit $Z \rightarrow a \gamma$ decays [36, 37]. In special, the limits imposed on $Z \rightarrow \gamma \gamma(\gamma)$ from the CDF [38] and the ATLAS [28] Collaborations should be taken into account for ALP masses in the 0.5 to $60 \mathrm{GeV}$ region. Taking the strongest limit from the ATLAS search for $Z$ bosons decaying into three photons [28], we have

$$
\frac{m_{Z}^{3}}{6 \pi \Gamma_{Z}}\left(1-\frac{m_{a}^{2}}{m_{Z}^{2}}\right)^{3} s_{W}^{2} c_{W}^{2}\left(\frac{k_{B B}}{\Lambda}-\frac{k_{W W}}{\Lambda}\right)^{2} \times B R(a \rightarrow \gamma \gamma)<2.2 \times 10^{-6},
$$

where $m_{Z}=91.18 \mathrm{GeV}$ and $\Gamma_{Z}=2.5 \mathrm{GeV}$ are the $Z$ mass and width, respectively. For $m_{a} \in[0.5,60] \mathrm{GeV}$ and $B R(a \rightarrow \gamma \gamma)$ from $1 \%$ to $50 \%$, this constraint implies $\left|\frac{k_{B B}}{\Lambda}-\frac{k_{W W}}{\Lambda}\right| \lesssim 10^{-4}$ to $10^{-5}$ that can be achieved for $k_{B B}, k_{W W} \lesssim 10^{-1}$ to $10^{-2}$ and $\Lambda \gtrsim 1 \mathrm{TeV}$.

The ATLAS Collaboration put limits on Higgs-ALP couplings from searches for $h \rightarrow \gamma \gamma$ for the ALP mass range of 100-400 MeV [39]. As we are going to discuss, we estimate, and also observe in the simulations, that resonances in two resolved photons can indeed be observed for $m_{a} \gtrsim 10 \mathrm{GeV}$, and the $0.5-10 \mathrm{GeV}$ mass region is a transition zone where photons from ALP decays begin to be resolved in the detectors. Therefore, we do not place Higgs to two photons constraints on the model once it is difficult to ascertain the validity of those constraints in this transition mass range.

Collider searches in diphotons and dijets look for high mass resonances and do not constrain the ALPs we consider in this work. Astrophysical and other low-energy experiments that hunt axions and axion-like particles have sensitivity to ALPs which are much lighter than the ones we are focusing on, i.e. in the 0.5 to $60 \mathrm{GeV}$ mass region. For a good account of constraints on masses and couplings of effective ALP models, see ref. [30].

In the section 5 , we show the relative number of parameter space points that survive the constraints imposed by eqs. (3.1)-(3.6). Now, we give details about the simulations and the search analysis.

\section{Simulation details and search analysis}

We simulated events for the signal $g g \rightarrow h \rightarrow a a \rightarrow g g \gamma \gamma$, leading to at least one isolated jet and one isolated photon, one or two isolated photons plus any number of isolated jets, and its main SM backgrounds: the prompt production $j \gamma$ with one additional jet, $j j \gamma$; 
the double photon production with one and two additional jets $j \gamma \gamma$, and $j j \gamma \gamma$; the dijet production $j j$, where the decay $\pi^{0} \rightarrow \gamma \gamma$ and other scalar mesons inside the jets produce one or two hard isolated photons. The Higgs decay to a gluon jet plus photons is forbidden by color charge conservation, while the three and four-body decays to $h \rightarrow g g \gamma$ and $h \rightarrow g g \gamma \gamma$ are suppressed by $\mathcal{O}(\alpha)$ and $\mathcal{O}\left(\alpha^{2}\right)$ couplings, respectively, in comparison to $h \rightarrow g g$. Note that initial state photon radiation does not occur in gluon fusion. Moreover, as we will discuss, the events are required to present narrow resonances compatible with ALP decays, which further suppress three, and four-body Higgs decays.

The simulations were performed at leading-order for the $13 \mathrm{TeV}$ LHC using MadGraph5 [40]. Parton hadronization and showering were simulated with Pythia8 [41], while detector effects were taken into account from Delphes3 [42].

The first difficulty we must overcome is the huge QCD $j j$ background which amounts to $\sim 10^{11}$ femtobarns at the $13 \mathrm{TeV}$ LHC. A hadronic jet contains pions and other mesons that might decay into hard and isolated photons that mimic the signal. For commonly adopted photon isolated criteria, one in every hundred jets, approximately, will look like an isolated photon which means that we should expect around $10^{7} \mathrm{fb}$ cross section of fake $j+\gamma$ from the $j j$ background, that turns out to be similar to the irreducible prompt photon background cross section. In order to reduce this potentially dangerous background source, we demand a tight photon isolation criteria inspired in refs. [28, 43]. A photon is considered isolated if particles with $p_{T}>0.5 \mathrm{GeV}$ inside a cone of $\Delta R=0.4$ centered around the photon 3-momentum do not deposit a total fraction of their transverse momentum above 0.05 of the photon $p_{T}$. Jets are reconstructed using the anti-kt algorithm with a radius parameter of $R=0.4$ with a minimum transverse momentum of $20 \mathrm{GeV}$. The tight isolation criterion guarantees that a small fraction of multijet events will produce an isolated photon making it easier to suppress the huge $j j$ background [43]. Our simulations show that these tight isolation criteria reduce the $j j$ background to less than $10 \%$ of the irreducible $j+\gamma$ background. $^{2}$ Nevertheless, we analyze the possible effect of the remaining $j j$ samples as a systematic uncertainty effect in the main background estimates. Also, this isolation requirement comes at the cost of suppressing ALP signals from ALPs with masses at the transition mass region where not too collimated ALP decays start to get resolved by the detectors.

Reconstructed photons are obtained from clusters of energy deposited in an electromagnetic calorimeter (ECAL) of finite granularity in the LHC detectors. When two particles hit a small area of the detectors, the signal might be interpreted as a single hit. The distance in the $\eta \times \phi$ plane between the particles from the ALP decay (which comes from a Higgs boson decay) is given by $\Delta R \sim \frac{2 m_{a}}{p_{T}} \leq \sqrt{(\Delta \phi)^{2}+(\Delta \eta)^{2}}$, where $p_{T}$ denotes the transverse momentum of the ALP, and the azimuth and rapidity resolutions of the LHC detectors are $\Delta \phi$ and $\Delta \eta$, respectively. Thus ALP masses up to $\sqrt{(\Delta \phi)^{2}+(\Delta \eta)^{2}} \times p_{T} / 2 \mathrm{GeV}$ lead to unresolved decay products, two photons form a single "photon-jet", and two gluons form a single hadronic jet. Adopting the default resolutions of Delphes3 in the rapidity

\footnotetext{
${ }^{2}$ We also checked that our simulations reproduce very well the $j+\gamma$ cross section obtained in ref. [43] with their isolation and selection criteria.
} 
region of $|\eta|<3.0, \Delta \phi=\Delta \eta=0.02$, and given that the bulk of events have $p_{T} \sim m_{h} / 2$ ( $m_{h}=125 \mathrm{GeV}$ is the Higgs boson mass), we should expect that most of the signal events are still collimated for ALP masses up to $\sim 1 \mathrm{GeV}$. As we discussed in section 3 , the ATLAS Collaboration puts limits on ALP signals for $m_{a}<0.4 \mathrm{GeV}$ from their search for $h \rightarrow \gamma \gamma$ with collimated ALP decays. Anyway, the ALP mass region of a few $\mathrm{GeV}$ is a transition region between the resolved and unresolved jets and photons signals. Collider searches for ALP signals into resolved and unresolved photon-jets have been studied, for example, in refs. [32, 44-46].

Because we are interested in the mass region where we expect to observe resolved and unresolved photons and jets, we adopted an inclusive search channel accepting at least one isolated jet and one isolated photon with

$$
\begin{aligned}
p_{T}^{j} & >30 \mathrm{GeV}, \quad\left|\eta_{j}\right|<2.4, \\
p_{T}^{\gamma} & >20 \mathrm{GeV}, \quad\left|\eta_{\gamma}\right|<2.4, \\
\Delta R_{j \gamma} & >0.4
\end{aligned}
$$

As we said, the background simulations included the prompt photon+jet production, $q \bar{q} \rightarrow g+\gamma$ and $g q \rightarrow q+\gamma$ taking into account all the QCD and QED hard contributions up to an extra jet and an extra photon. We can classify these background sources in four classes: (1) $j \gamma$, (2) $j j \gamma$, (3) $j \gamma \gamma$, and (4) $j j \gamma \gamma$. We found that contributions from $Z(\rightarrow j j) \gamma(\gamma), W(\rightarrow j j) \gamma(\gamma)$ and Higgs decays to photons and gluon jets are negligible after imposing cuts and isolation criteria. Photons from fragmentation or radiation from hadronic taus are not sufficiently isolated and can also be neglected. We estimate that those backgrounds do not contribute more than a few events after $3 \mathrm{ab}^{-1}$. ALP pair production and associated ALP plus photons and jets [47] could also mimic the signal from Higgs decays to ALPs, but, as we will discuss, the photons and jets are required to reconstruct the Higgs mass peak, which effectively suppresses those contributions to the signal. Now, we discuss the selection criteria to detect the signals.

\subsection{Selection criteria - Higgs-ALP spectroscopy}

The most discerning features of the signals are their invariant mass spectra, which could be thought of as distinct spectral lines. In figure 1, we display the invariant mass distribution of $j j$ pairs and the leading $p_{T}$ jet $(J)$ mass at the upper row, and the $\gamma \gamma$ and the $j(j)+\gamma(\gamma)$ masses at the lower row.

The $M_{j j \gamma \gamma}$ shows the jets plus photons invariant mass. This variable is computed with up to two leading photons and two leading jets, also comprising events with just one jet and one photon solely. From the lower panels of figure 1, we can see a clear peak at the Higgs mass in $M_{j j \gamma \gamma}$ from small ALP masses up to $10 \mathrm{GeV}$. For higher ALP masses, the $j(j)+\gamma(\gamma)$ invariant mass does not peak right at the Higgs mass anymore. This is due to the tight photon isolation criteria needed to suppress the multijet QCD background, which makes the identification of two isolated photons rarer than only one photon. Consequently, for heavier ALP masses, where two resolved photons and jets were expected, many events turn out to have just one tightly isolated photon, and the combined masses of the final 

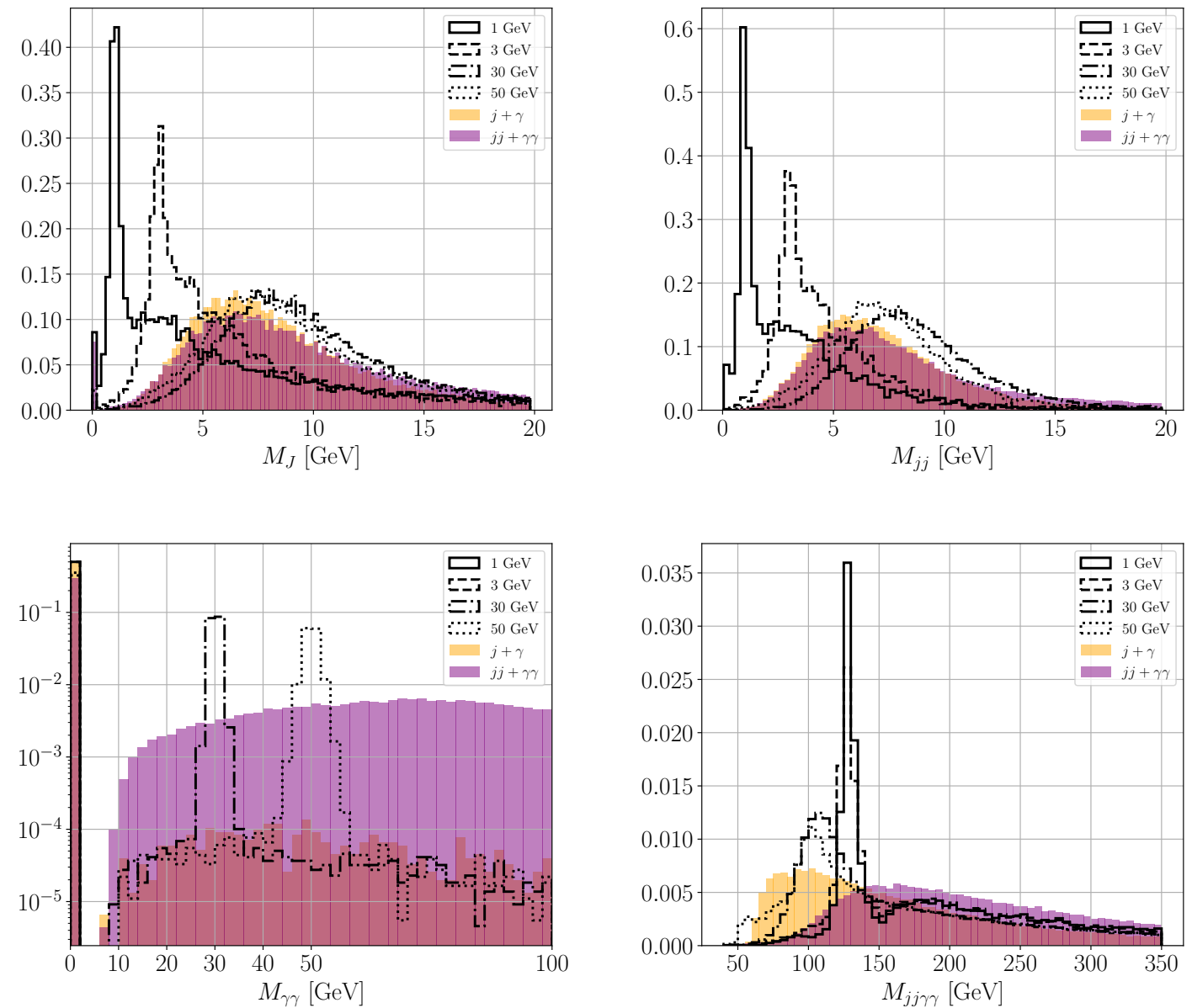

Figure 1. The normalized invariant mass distributions of photon pairs (lower left), jet pairs (upper right), the mass of the leading $p_{T}$ jet, $J$ (upper left), and the mass of $j(j)+\gamma(\gamma)$ pairs (lower right). We display the distributions for ALP masses of $1,3,30$, and $50 \mathrm{GeV}$ and for the $j+\gamma$ and $j j \gamma \gamma$ backgrounds.

state particles do not add to the Higgs mass. On the other hand, for lighter masses, the identified leading jet and photon come from collimated pairs and reconstruct the ALPs and the Higgs.

In those cases where two isolated photons can be identified in the event, we observe a peak in the $\gamma \gamma$ mass, as we see in the lower left panel of figure 1. However, if the event contains just one isolated photon, even if it is a photon-jet composed of two collimated photons, it is interpreted as a single photon of null mass. That is why we see a populated bin at $0 \mathrm{GeV}$ in the $M_{\gamma \gamma}$ distribution.

In events where just one isolated photon is detected, we switch to one- or two-jet masses to observe the ALP resonance, as we can see in the upper panels of figure 1. In those events where just one isolated jet can be identified, we observe that the jet mass exhibits a peak for lighter masses (see the upper left panel of figure 1), once the reconstructed jet will contain the two jets of the event collimated into a single fat jet. This explains the similarity between 
the $M_{J}$ distribution and $M_{j j}$, which is the mass of up to two leading jets, including the events with just one identified jet. In practice, we could use just $M_{j j}$ to look for light ALP peaks in cases where at least one isolated jet is present, but we also used $M_{J}$ to place cuts. The difficulty arising from the tight photon isolation criteria in identifying the Higgs and the ALP resonances calls for an automatic and optimized way to identify the resonances and signal-rich regions of the feature space in the search for ALPs with unknown masses.

Along with the invariant masses just described, we compound our events representation with the four additional distributions of figure 2. The upper row displays the transverse momentum of the $\gamma(\gamma)$ and $j(j)$ pair of the events at the left and right panels, respectively. In events where just one photon or jet is identified, the transverse momentum of the pair is substituted by the particle $p_{T}$. The lower panels show two other useful distributions, the number of tracks of the event at the left panel, and the $\cos \theta_{j \gamma}^{*}=\frac{1}{2} \tanh \left(\left(\eta_{j}-\eta_{\gamma}\right) / 2\right)$ at the right panel. This later variable effectively measures the cosine of the angle between the leading photon and the leading jet directions. For processes where the photon and the jet are collimated, $\theta^{*}$ tends to vanish. By their turn, signals from light ALP masses exhibit fewer detector tracks than backgrounds, whilst larger masses have a slightly larger number of tracks.

In the gluon fusion process, the Higgs production cross section at the $13 \mathrm{TeV}$ LHC is $48.5 \mathrm{pb}$ [40]. The maximum Higgs to ALP branching ratio allowed by the LHC constraint of eq. (3.5) is $32.2 \%$. In contrast, the maximum branching fraction to $j j \gamma \gamma$ is $2 \times(0.5)^{2}=0.5$, producing a maximum total branching ratio of around $16 \%$, representing a 7.8 pb signal at most. The dominant irreducible background, $j \gamma$, by its turn, has a cross section of $3.02 \times 10^{4}$ $\mathrm{pb}$, including an extra jet contribution. The production cross section of the dominant reducible background of QCD jet pair production is huge, amounting to $2.1 \times 10^{8} \mathrm{pb}$ after the basic cuts of eq. (4.1). The $j j \gamma \gamma$ cross section is $24.3 \mathrm{pb}$ after the basic cuts. As we discussed, the first step in reducing the backgrounds is to impose the tight photon isolation criteria that suppresses the $j j$ background, otherwise, observing light ALP signals would be impossible. The isolation requirements affect most of the light ALP signals, reaching a minimum efficiency of $5 \%$ for $m_{a}=3 \mathrm{GeV}$. On the other hand, they allow less than 1 in every $10^{4}$ events of jet pair production, approximately, bringing the $j j$ contamination to a fraction of the $j \gamma$ background. The photon isolation efficiency for $j \gamma$ and $j j \gamma \gamma$ backgrounds are $58 \%$ and $77 \%$, respectively.

In order to automatically adapt the selection strategy to different ALP masses and optimize the signal significance, we used an optimization algorithm based on Tree Parzen Estimation (TPE) to search for the best cuts on the eight variables of figures 1 and 2 with the aid of Hyperopt $[48,49]$. Five thousand searches for each mass were performed, seeking to maximize the statistical significance calculated from

$$
N_{\sigma}=\frac{S}{\sqrt{B+\left(\varepsilon_{B} B\right)^{2}}},
$$

where $S(B)$ is the number of signal(total background) events for a given integrated luminosity, and $\varepsilon_{B}$ represents a systematic uncertainty in the total background rate. The systematic uncertainty was fixed at 0.01 to perform the searches so that the algorithm 

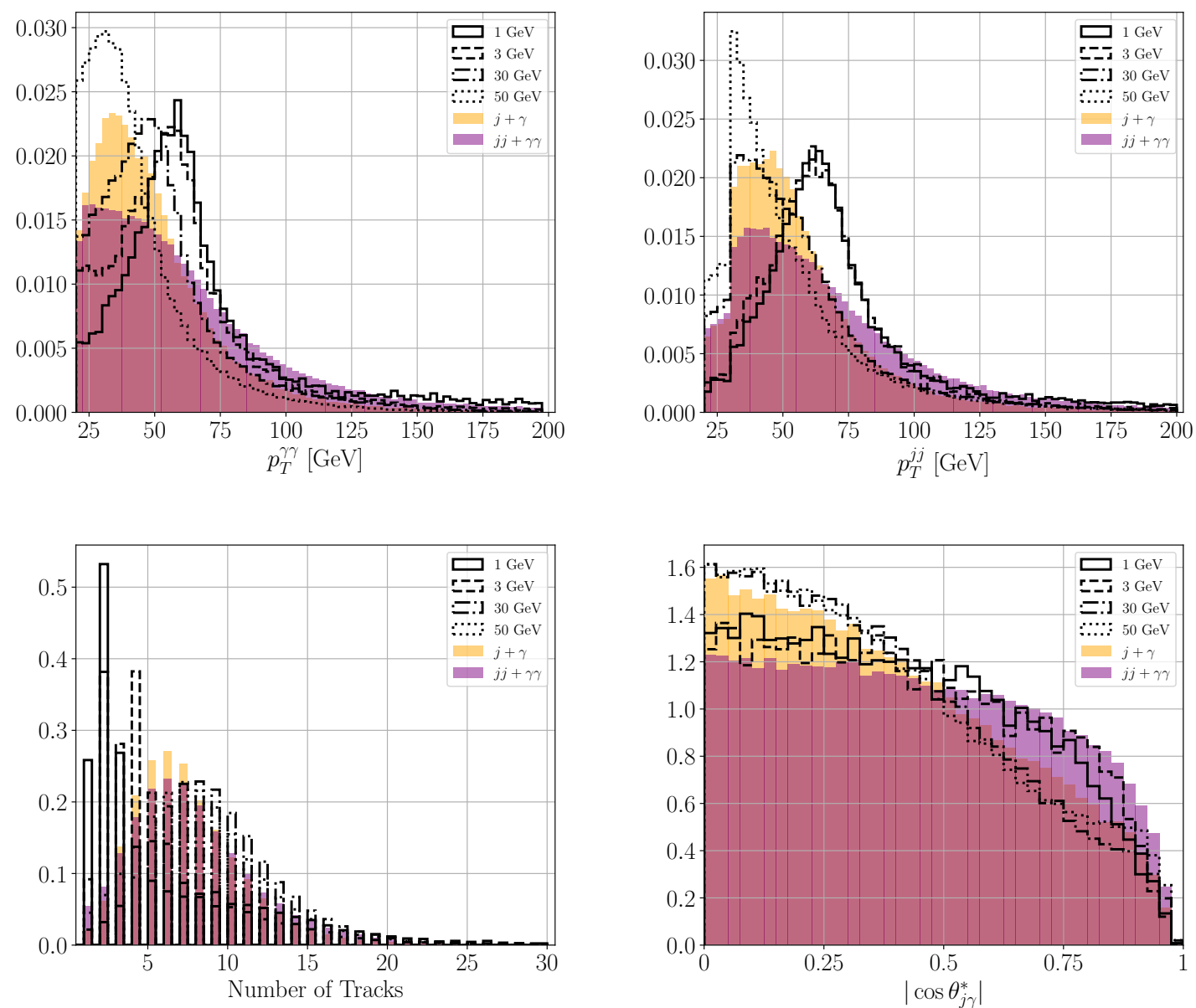

Figure 2. The $\gamma(\gamma)$ transverse momentum (upper left), $j(j)$ transverse momentum (upper right), the number of tracks of the leading jet (lower left), and $\cos \theta_{j \gamma}^{*}$ (lower right). We display the distributions for ALP masses of $1,3,30$, and $50 \mathrm{GeV}$ and for the $j+\gamma$ and $j j+\gamma \gamma$ backgrounds. All distributions are normalized to unity.

learns to tame the systematics, raising the $S / B$ ratio [49]. As an example, we show, in eq. (4.3), the cuts that maximize the signal significance metric of eq. (4.2) for an ALP mass of $10 \mathrm{GeV}$

$$
\begin{aligned}
0.4 & <M_{J}<12.4 \mathrm{GeV}, & 0.9 & <M_{j j}<13.6 \mathrm{GeV}, \quad\left|M_{j j \gamma \gamma}-m_{h}\right|<9.4 \mathrm{GeV}, \\
66 & <p_{T}^{\gamma \gamma}<237.5 \mathrm{GeV}, & 37 & <p_{T}^{j j}<74 \mathrm{GeV}, \\
14 & <n_{\text {tracks }}<33, & 0 & <\left|\cos \theta_{j \gamma}^{*}\right|<0.6 .
\end{aligned}
$$

In this case, the algorithm learned that searching for a peak in the leading jet mass and $j j$ mass distributions is more efficient than in the $\gamma \gamma$ spectrum and, indeed, the windows where the cuts are placed in those distributions contain the mass value of $10 \mathrm{GeV}$. Moreover, note that the $p_{T}^{\gamma \gamma}$ and $p_{T}^{j j}$ cuts focus around $m_{h} / 2$ where these distributions should be peaked for the signals. This cut strategy leads to a signal significance of $20.8 \sigma$, after 3 $\mathrm{ab}^{-1}$, at a point of the parameter space where $C_{a h}^{\mathrm{eff}}=1 \mathrm{TeV}^{-2}$ and $B R(h \rightarrow a a)=21.7 \%$. The overall efficiencies for the signal, $j+\gamma$, and $j j+\gamma \gamma$ backgrounds read $2 \times 10^{-4}$, 
$1.4 \times 10^{-7}$, and $2.8 \times 10^{-6}$, respectively. The subdominant backgrounds of $Z(\rightarrow j j) \gamma(\gamma)$, $W(\rightarrow j j) \gamma(\gamma), h(\rightarrow \gamma \gamma)+j, h(\rightarrow j j)+\gamma$, and $h \rightarrow \tau_{h} \tau_{h}$ all fail to reconstruct the Higgs resonance with photons and jets and also/or the ALP resonances and turn out to be easily vetoed. Other masses present similar combined cut and identification efficiencies.

Such small cut efficiencies pose a problem to the analysis once it is very time-consuming to generate sufficiently many Monte Carlo events for a reliable estimate. We overcome this difficulty by generating a large number of synthetic events using a well-known data augmentation machine learning technique to tackle imbalanced datasets. In both machine learning studies and phenomenological analysis as ours, classifying events with good accuracy and small false positive/negative rates demands balanced datasets. One of those techniques is the Synthetic Minority Oversampling TEchnique [50], or SMOTE, for short, based on a straightforward idea. First, an event is randomly chosen from the dataset and its $k$ nearest neighbors are found, then one of these neighbors is randomly chosen. After determining those two events, a line is drawn connecting them in the representation space of the event (the space of kinematic and other variables of the tabulated event). Finally, a point in this line between those two events is randomly picked according to some distribution. In SMOTE, this distribution is uniform. In this work, we actually used the Adaptive Synthetic Sampling Method [51], or ADASYN, that chooses a new point according to a local density distribution of points, thus trying to mimic, or adapt, to the true local density of points in the vicinity of the chosen event that seeds the generation of the synthetic point. ${ }^{3}$

To generate synthetic data, we firstly produced a few million MC samples from MadGraph for signals and backgrounds. After isolation, identification, and basic cuts have been imposed, 10 to 100 thousand events survive depending on the process. These samples are then used to seed the generation of synthetic samples iteratively. To do so, we split the MC samples into two randomly chosen disjoint sets where the minority one contains a fraction $\alpha$ of the total number of samples. The algorithm then produces several synthetic samples to turn the size of two sets equal, that is it, $1-2 \alpha$ times the size of the original MC event samples. We repeat this procedure until we get the desired number of samples. In figure 3 , we show the $M_{j j \gamma \gamma}$ distribution for the signal (left panel) and the $j+\gamma$ (right panel) background. Just like this kinematic variable, we checked that synthetic and original samples generated by MadGraph display very similar shapes for all the other variables. Moreover, we also observed that the algorithm could populate sparse portions of the feature space, effectively producing events in the tails of the distributions and helping to estimate the cut efficiencies better.

With this technique, we generated $\mathcal{O}\left(10^{6}\right)$ events to each background component and the signals. We also generated around $10^{7} j j$ events to check its selection efficiency after imposing the tight isolation criteria. We used all these events to tune the cut strategy for several ALP masses from 0.5 to $60 \mathrm{GeV}$, fixing a $1 \%$ systematic uncertainty in the total background rate as discussed earlier. Before we present the results of our analysis, let us investigate the points of the parameter space considered in this work that satisfy the constraints of section 3 .

\footnotetext{
${ }^{3}$ Neural networks based data augmentation in high energy collision simulations have been addressed, for example, in refs. [52-54]. A detailed studied to compare the performance of our proposed $k \mathrm{NN}$ approach to other ones is due.
} 

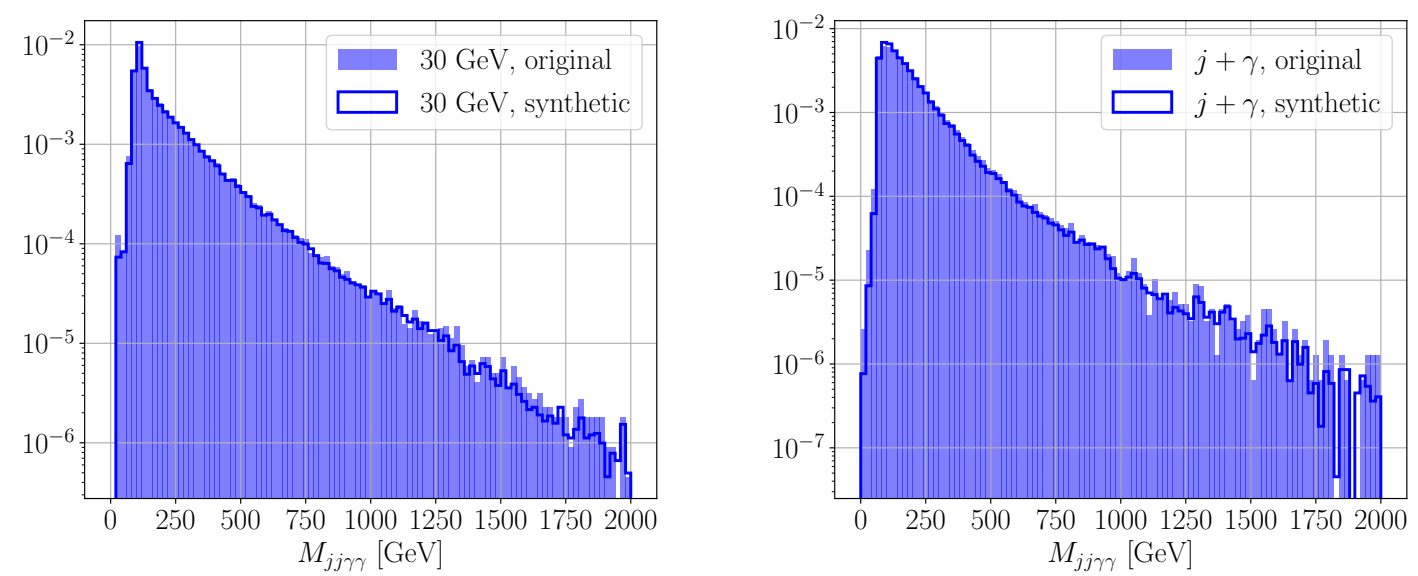

Figure 3. Comparison between the original MC events generated by MadGraph (blue shaded histograms) and the synthetic ones generated with the ADASYN method (solid blue). We display the normalized $M_{j j \gamma \gamma}$ distribution for the ALP signal $\left(m_{a}=30 \mathrm{GeV}\right)$ at the left panel, and for the $j+\gamma$ background at the right panel.

\section{Scanning the parameter space}

Before proceeding to the results section, we need to estimate how many points of the model's parameter space could eventually be probed at the LHC. For that goal, we scan a relevant portion of the parameter space defined by

$$
\begin{aligned}
& \frac{k_{G G}}{\Lambda} \in\left[10^{-3}, 4 \pi\right] \mathrm{TeV}^{-1}, \quad \frac{k_{\gamma \gamma}}{\Lambda} \in\left[10^{-3}, 4 \pi\right] \mathrm{TeV}^{-1}, \frac{C_{a h}^{\mathrm{eff}}}{\Lambda^{2}} \in\left[10^{-4}, 4 \pi\right] \mathrm{TeV}^{-2}, \\
& m_{a} \in[0.5,62.5] \mathrm{GeV} .
\end{aligned}
$$

Although we have chosen a different and more convenient basis to parametrize our model, some of the constraints of section 3 can only be translated to the original parameters contained in the Lagrangian of eq. (2.1). For this reason, we first draw samples from the original parameters of eq. (5.1), apply the constraints of eqs. (3.1)-(3.6), and then map those points that passed the requirements onto our chosen parameter space: $C_{a h}^{\text {eff }} / \Lambda^{2}$, $B R(a \rightarrow \gamma \gamma)$ and the ALP mass, $m_{a}$, as discussed in section 2 .

After the random draw of $2 \times 10^{6}$ points from log-uniform distributions and imposing all the constraints from eqs. (3.1) to (3.6), around $20 \%$ of the points passed all of those constraints. Eq. (3.5), limiting the largest branching ratio of the Higgs boson into new modes, and eq. (3.6), that constrains photonic decays of the $Z$ boson, are the harder ones.

In figure 4, left panel, we show the result of the scan displaying the density of the parameters effectively used to parametrize the model for points that evade the constraints imposed in the $C_{a h}^{\mathrm{eff}} / \Lambda^{2}$ versus $m_{a}$ plane, the parameters in which we are interested in displaying the results. We see that eq. (3.5) prohibits points above the dashed line, but weakens as $m_{a}$ approaches $m_{h} / 2$. As a result, a slightly larger concentration of points occurs for heavier masses near $m_{h} / 2$, yet we have a roughly uniform density of points in the whole ALP mass range. 

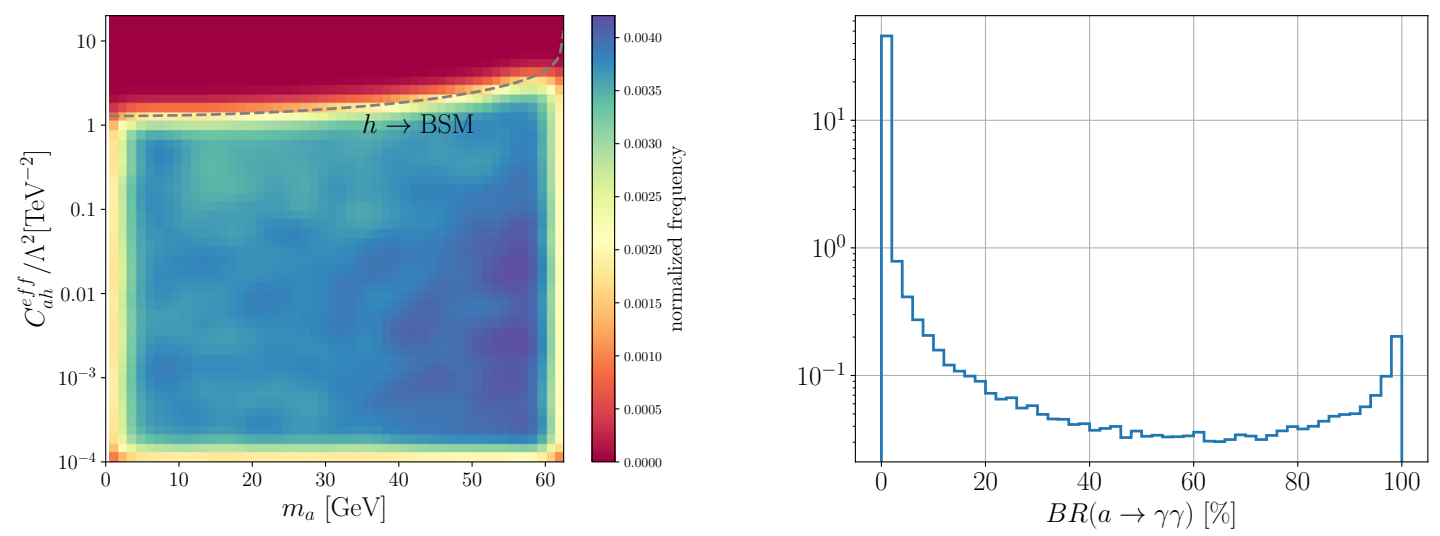

Figure 4. At the left panel, we show the density of points of the parameter space eq. (5.1) after imposing the collider constraints of section 3 in the $C_{a h}^{\text {eff }} / \Lambda^{2}$ versus $m_{a}$ plane. Cooler regions have a larger density of points. The dashed line represents the constraint from eq. (3.5). At the right panel, we plot the histogram of the normalized frequency of valid points for a given ALP branching ratio to photons.

At the right panel of figure 4, we display the density of points in terms of the branching ratio of the ALP into photons. The majority of points are found for very small values of the branching ratios, but we can also find points where $B R(a \rightarrow \gamma \gamma) \approx 50 \%$. From eq. (2.2), the branching ratio of the ALP into photons can be expressed as $1 /\left(1+8 k_{G G}^{2} / k_{\gamma \gamma}^{2}\right)$, and the ratio $k_{G G}^{2} / k_{\gamma \gamma}^{2}$ can assume values from $10^{-4}$ up to $10^{4}$ when parameters vary as in eq. (5.1). This ratio is evenly distributed around unit, giving the branching ratio distribution an almost symmetric shape. The constraints from eqs. (3.3), (3.4) and (3.6), however, eliminate those points with large $B R(a \rightarrow \gamma \gamma)$, and only those ones where the Higgs-ALP coupling is too small and/or $k_{B B} \approx k_{W W} \gg k_{G G}$ can have a large ALP to photons decay rate. Now, we present the results of our analysis.

\section{Results}

After tuning the cuts to reach the maximum sensitivity for each ALP mass, we used the cut efficiencies to estimate the signal significance by computing the branching ratios at a given point of the parameter space. The signal cut efficiency depends only on the ALP masses, which modify the kinematic distributions. Our goal is to evaluate the sensitivity of the $j(j)+\gamma(\gamma)$ channel and compare it against the $j j \gamma \gamma$ and $\gamma \gamma \gamma \gamma$ channels given by eqs. (3.3) and (3.4), respectively, in the $C_{a h}^{\text {eff }} / \Lambda^{2}$ versus $m_{a}$ plane.

We show in figure 5 , the regions of the $C_{a h}^{\text {eff }} / \Lambda^{2}$ versus $m_{a}$ plane which can be probed at $95 \%$ CL in the $j(j)+\gamma(\gamma)$ channel in four different systematic error scenarios: statistics domination $\left(\varepsilon_{B}=0\right)$ and $1 \%$ systematics at the left and right upper panels, respectively, and $5 \%$ and $10 \%$ at the left and right lower panels, respectively. Dot-dashed lines in that figure represent points of the parameter space for which $B R(a \rightarrow \gamma \gamma)=1 \%$, while the solid curves represent the case where $B R(a \rightarrow \gamma \gamma)=50 \%$. We found that systematic uncertainties dominate the errors for $\varepsilon_{B} \gtrsim 3 \%$. As a consequence, if the background rates 

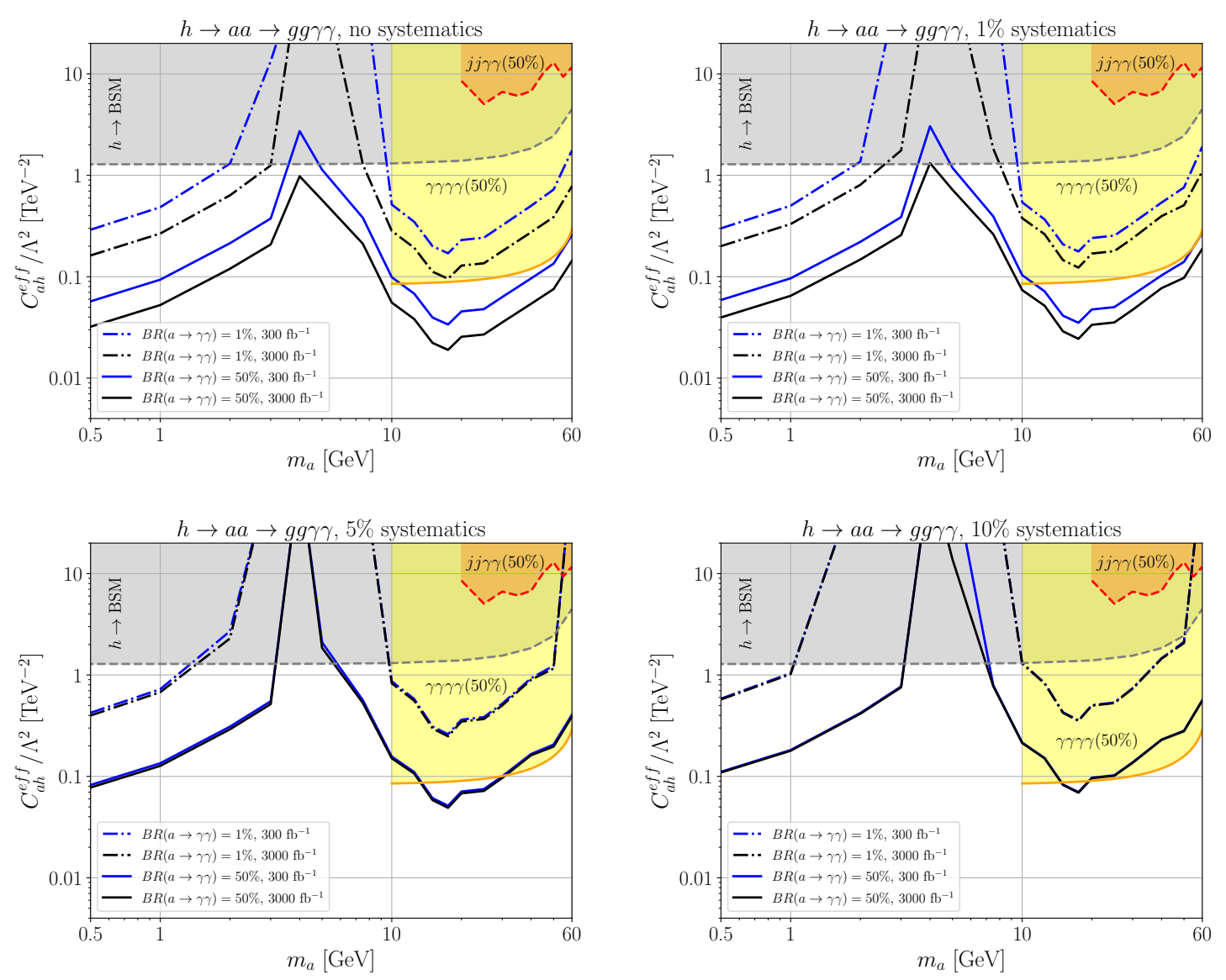

Figure 5. The $95 \% \mathrm{CL}$ limits on the effective ALP-Higgs coupling (in $\mathrm{TeV}^{-2}$ ) versus the ALP mass (in $\mathrm{GeV}$ ). The orange shaded area denotes the limits from eq. (3.3), while the yellow shaded area represents the limits from eq. (3.4), assuming $B R(a \rightarrow \gamma \gamma)=50 \%$ in both cases. The blue(black) lines represent the limits obtained in the $j(j)+\gamma(\gamma)$ channel assuming $300(3000) \mathrm{fb}^{-1}$ of data, while the solid(dot-dashed) curves represent the case where branching ratio of the ALP to photons is $50 \%(1 \%)$. We show four different systematics scenarios: $\varepsilon_{B}=0,1,5,10 \%$. The gray shaded region $(h \rightarrow \mathrm{BSM})$ is excluded, at $95 \% \mathrm{CL}$, by the LHC constraint on the Higgs total width, eq. (3.5).

can be precisely determined, we should expect to observe improvements of statistical limits with increasing luminosity, otherwise, the limits for 300 and $3000 \mathrm{fb}^{-1}$ will look very similar as we see in the lower panels of figure 5 .

As expected, the regions of the parameter space that can be probed for a fixed luminosity improve as $B R(a \rightarrow \gamma \gamma)$ approaches $50 \%$. The maximum sensitivity occurs for ALP masses close to 0.5 and $20 \mathrm{GeV}$, two well distinct regimes where the majority of events contain totally unresolved $j+\gamma$ pairs and totally resolved $j j+\gamma \gamma$ pairs, respectively, reaching $C_{a h}^{\mathrm{eff}} / \Lambda^{2} \approx 0.02 \mathrm{TeV}^{-2}$. Even for the high systematic error scenario of $10 \%$, an effective ALP-Higgs coupling of order $0.1 \mathrm{TeV}^{-2}$ can be excluded at the $95 \% \mathrm{CL}$. On the other hand, unless the systematics are well controlled, a mass gap between 2 and $5 \mathrm{GeV}$, approximately, might remain unreachable at the $13 \mathrm{TeV}$ LHC. This is a mass region where the overall signal efficiency, including isolation and kinematic cut efficiencies, reaches a 
minimum, around $4 \mathrm{GeV}$. We also checked that this region produces the minimum number of totally resolved $j j+\gamma \gamma$ events for which the selection algorithm more easily identifies the mass peaks, and the number of totally unresolved $j+\gamma$ is dropping. This is precisely the mass region where the unresolved pairs become resolved but not sufficiently isolated to escape our selection requirements. A possible alternative to raising the signal efficiency for that mass gap is changing the definition of photon jets to become more inclusive, as in ref. [32]. However, it is necessary to evaluate the impact on the rejection efficiency of the QCD multijet background. Finally, the very light end of the former mass gap region, between 0.5 and $2 \mathrm{GeV}$, can be probed in the $j(j)+\gamma(\gamma)$ channel, contrary to the four photons and the fully resolved $j j \gamma \gamma$ channels.

In the mass region where $m_{a}>10 \mathrm{GeV}$, we display the current constraint from the ATLAS search for $h \rightarrow \gamma \gamma \gamma \gamma$, eq. (3.4). This search was performed at the $8 \mathrm{TeV} \mathrm{LHC}$ after $20.3 \mathrm{fb}^{-1}$, so we expect that future reevaluations of these limits get significantly stronger. These results, however, are very sensitive to $B R(a \rightarrow \gamma \gamma)$. In figure 5 , we display the region of the parameters excluded at 95\% CL by this search channel if $B R(a \rightarrow \gamma \gamma)=50 \%$ (solid yellow line). The $1 \%$ case does not constraint the region of the parameters displayed in the plot. We basically learn that $j(j)+\gamma(\gamma)$ is competitive if the ALP branching ratio into photons is small, disregarding the level of systematics affecting the jets plus photons channel. On the other hand, $h \rightarrow \gamma \gamma \gamma \gamma$ will remain as the best option to constrain models where the ALP branching ratio into photons is large if $m_{a}>10 \mathrm{GeV}$. As we anticipated, the current limits from eq. (3.3) do not harm the particular effective model that we are considering in this work. This experimental search involved $36.7 \mathrm{fb}^{-1}$ of $13 \mathrm{TeV}$ LHC data [55], and it is also expected to improve in the future.

Comparing the figure 5 against the left panel of figure 4, we see that a large number of points that evade the collider constraints can be probed in the $j(j)+\gamma(\gamma)$ channel in the whole region between 0.5 and $60 \mathrm{GeV}$. From the right panel of figure 4, we learned that most points have $B R(a \rightarrow \gamma \gamma)$ close to $1 \%$ and that a branching ratio close to $50 \%$, for which the sensitivity is maximum, is rarer. Nevertheless, we found that small branching ratios for $a \rightarrow \gamma \gamma$ can be probed even with $300 \mathrm{fb}^{-1}$ if the systematic uncertainties could be kept under control.

In figure 6 , we show the regions of the $C_{a h}^{\text {eff }} / \Lambda^{2}$ versus $m_{a}$ where $N_{\sigma} \geq 5 \sigma$ for two systematic error scenarios, $1 \%$ and $10 \%$ at the left and right panels, respectively. Assuming $B R(a \rightarrow \gamma \gamma)=1 \%$, we see that little room is left for discovery that has not been excluded by the collider constraints in the low ALP mass region, however, there is a sizeable portion of the parameter space not excluded if $m_{a}>10 \mathrm{GeV}$. The picture changes for $B R(a \rightarrow$ $\gamma \gamma)=50 \%$, now a discovery is more likely in the mass region of $m_{a}<10 \mathrm{GeV}$, even for $10 \%$ systematics.

\section{Conclusions and prospects}

If axion-like particles exist, they should interact with the SM Higgs boson in an extended scalar sector. The Higgs-ALP coupling can be directly probed in decays of the Higgs into ALP pairs. Many possibilities exist to explore the yields of the ALP decays in different 

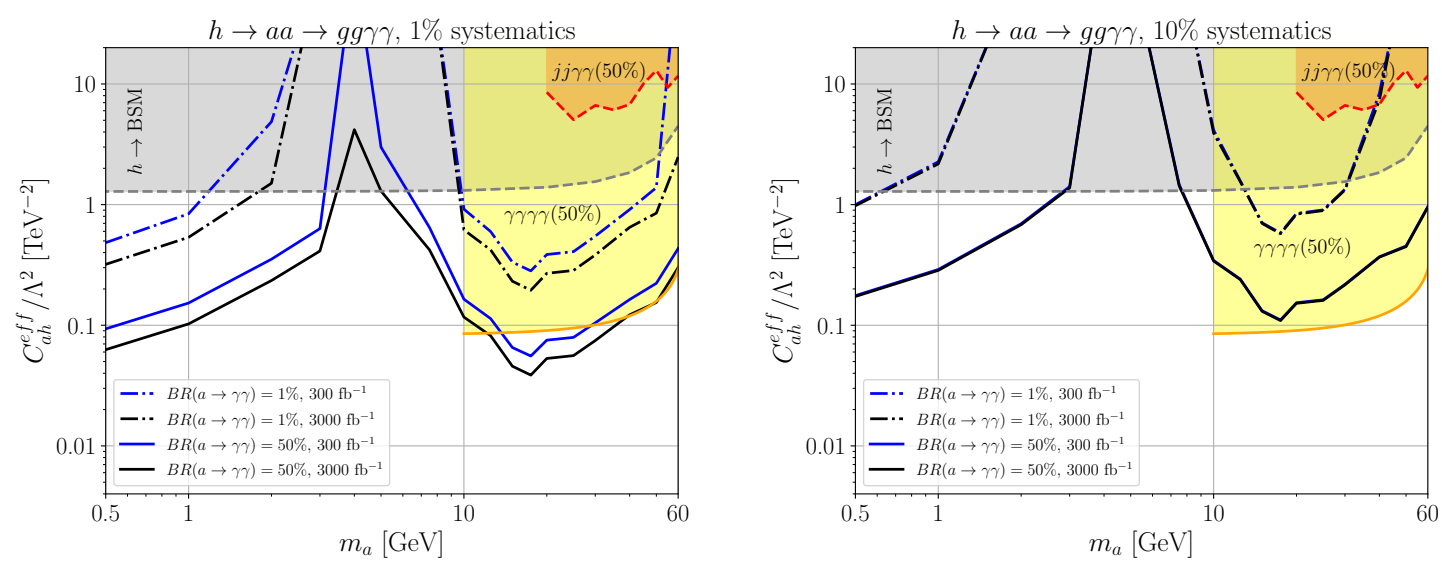

Figure 6. The region (above the lines) in the effective ALP-Higgs coupling (in $\mathrm{TeV}^{-2}$ ) versus the ALP mass (in GeV) where a discovery $(5 \sigma)$ of $h \rightarrow a a \rightarrow j(j)+\gamma(\gamma)$ is possible. The orange shaded area represents the excluded region from eq. (3.3), while the yellow shaded one shows the limits from eq. (3.4), assuming $B R(a \rightarrow \gamma \gamma)=50 \%$ in both cases. The blue(black) lines bound the $5 \sigma$ regions obtained in the $j(j)+\gamma(\gamma)$ channel assuming $300(3000) \mathrm{fb}^{-1}$ of data, while the solid(dotdashed) curves represent the case where the branching ratio of the ALP to photons is $50 \%(1 \%)$. We show two different systematic error scenarios: $\varepsilon_{B}=1,10 \%$. The gray shaded region $(h \rightarrow \mathrm{BSM})$ is excluded, at $95 \%$ CL, by the LHC constraint on the Higgs total width, eq. (3.5).

decay channels. An almost minimal approach assumes that the new scalar couples at least with gluons and photons, besides the Higgs bosons, and parametrizes the signals with fewer parameters, namely, the branching ratio of ALPs to photons, the Higgs-ALP coupling, and the ALP mass. The SM can be extended in many ways to account for new scalars with the properties of an ALP. Hence, an effective field theory model is suitable for a general parametrization of those possibilities assuming that, apart from the ALP, all the new particles are heavy enough to be integrated out, validating the effective field theory approach.

The Higgs-ALP coupling has mainly been searched for at colliders in processes with final state photons or leptons. These searches, however, lose sensitivity as soon as the ALP decays to hadrons are allowed, unless a large branching ratio to photons or leptons is assumed. In this work, we showed that the channel $p p \rightarrow h \rightarrow a a \rightarrow j(j)+\gamma(\gamma)$ might probe Higgs-ALP couplings in regions of the parameter space where the photons channels cannot, especially in the mass region of 0.5 to $10 \mathrm{GeV}$. Our analysis automatizes the cut strategy by searching for Higgs and ALP resonances in various jet and photon masses combinations in resemblance to spectroscopy characterization of material composition. This machine learning of the kinematic cuts to select signals enables us to place limits and estimate discovery prospects across the whole ALP mass region from $0.5 \mathrm{GeV}$, right after the hadronic decays are open, until $60 \mathrm{GeV}$, the Higgs to ALPs decay threshold.

We found that if the systematic uncertainties in the background rates can be controlled, the whole ALP mass region from 0.5 to $60 \mathrm{GeV}$ can be probed at 95\% CL even assuming a $1 \%$ ALP to photons branching ratio after $300 \mathrm{fb}^{-1}$ of data. With $3000 \mathrm{fb}^{-1}$ and 
$B R(a \rightarrow \gamma \gamma)=50 \%$, effective Higgs-ALP couplings down to $0.02 \mathrm{TeV}^{-2}$ can be excluded at $95 \%$ CL for ALP masses close to 0.5 and $20 \mathrm{GeV}$ but the $h \rightarrow a a \rightarrow \gamma \gamma \gamma \gamma$ might be a better option to probe the 10 to $60 \mathrm{GeV}$ mass range. A scan of the model's parameter space showed that the majority of the points that evade collider constraints could actually be probed in this proposed channel. Discovery is possible in scenarios where the systematic uncertainties are under control, and the ALP branching ratio into photons is sizeable in the mass range of 0.5 to $10 \mathrm{GeV}$, except for the $2-5 \mathrm{GeV}$ region. Overall, the $j(j)+\gamma(\gamma)$ channel is a better option than $h \rightarrow a a \rightarrow \gamma \gamma \gamma \gamma$ if the branching ratio of the ALP to photons is small and/or if the ALP is lighter than $10 \mathrm{GeV}$.

An important ingredient of our analysis is the suppression of the reducible $j j$ background, which produces isolated photons from neutral mesons decays. For that goal, a tight photon isolation requirement is necessary, but at the cost of also suppressing the signals for ALP masses from 2 to $5 \mathrm{GeV}$, a mass region where the photons and jets from the collimated ALP decays start to get resolved by the detectors. For masses larger than $\sim 10 \mathrm{GeV}$, the number of events with more than one isolated jet and photon increases, and we can search for the $h \rightarrow a a$ with a more inclusive topology. If the branching ratio of ALP to photons is large enough, the $j j+\gamma \gamma$ might provide tighter constraints than the four photons channel in the 10 to $60 \mathrm{GeV}$ region. Scenarios with masses lighter than $10 \mathrm{GeV}$ can be better probed when the ALP decays are collimated and lead to a $j+\gamma$ pair, which happens more efficiently in the 0.5 to $2 \mathrm{GeV}$ region. Overall, if systematic uncertainties could be tamed, the Higgs-ALP coupling can be probed at the HL-LHC in the whole mass region from 0.5 t0 $60 \mathrm{GeV}$.

Alternative Higgs production modes may not demand tight photon and jet isolation criteria. For example, in associated $Z h$ and $W h$ production [17], charged leptons and/or missing energy would hardly be mimicked by QCD $j j$ events, although other sources of backgrounds would come into play. The vector boson fusion channel also has good perspectives [29] and could benefit from a smart cut selection strategy. Finally, in this work, we did not attempt to tune the photon and jet isolation requirements once it is too computationally expensive, but at the analysis level, relaxing or tightening those criteria as the ALP mass varies would probably help to enhance the LHC sensitivity to Higgs-ALP interactions.

\section{Acknowledgments}

The works of A. A. and A.G. D. are supported by the Conselho Nacional de Desenvolvimento Científico e Tecnológico (CNPq), under the grants 307265/2017-0 and 306636/20166, respectively. D.D. L. acknowledges the financial support from the Coordenação de Aperfeiçoamento de Pessoal de Nível Superior - Brasil (CAPES) - 23 Finance Code 001. We also thank Julio Leite for reading the manuscript. 
Open Access. This article is distributed under the terms of the Creative Commons Attribution License (CC-BY 4.0), which permits any use, distribution and reproduction in any medium, provided the original author(s) and source are credited.

\section{References}

[1] Particle Data Group collaboration, Review of Particle Physics, PTEP 2020 (2020) 083C01 [INSPIRE].

[2] J.E. Kim and G. Carosi, Axions and the Strong CP Problem, Rev. Mod. Phys. 82 (2010) 557 [Erratum ibid. 91 (2019) 049902] [arXiv:0807.3125] [INSPIRE].

[3] J. Jaeckel and A. Ringwald, The Low-Energy Frontier of Particle Physics, Ann. Rev. Nucl. Part. Sci. 60 (2010) 405 [arXiv: 1002. 0329] [INSPIRE].

[4] A. Ringwald, Exploring the Role of Axions and Other WISPs in the Dark Universe, Phys. Dark Univ. 1 (2012) 116 [arXiv:1210.5081] [INSPIRE].

[5] D.J.E. Marsh, Axion Cosmology, Phys. Rept. 643 (2016) 1 [arXiv:1510.07633] [INSPIRE].

[6] L. Di Luzio, M. Giannotti, E. Nardi and L. Visinelli, The landscape of QCD axion models, Phys. Rept. 870 (2020) 1 [arXiv: 2003.01100] [InSPIRE].

[7] P. Agrawal et al., Feebly-Interacting Particles:FIPs 2020 Workshop Report, arXiv:2102.12143 [INSPIRE].

[8] M. Bauer, M. Heiles, M. Neubert and A. Thamm, Axion-Like Particles at Future Colliders, Eur. Phys. J. C 79 (2019) 74 [arXiv:1808.10323] [inSPIRE].

[9] R.D. Peccei and H.R. Quinn, CP Conservation in the Presence of Instantons, Phys. Rev. Lett. 38 (1977) 1440 [INSPIRE].

[10] R.D. Peccei and H.R. Quinn, Constraints Imposed by CP Conservation in the Presence of Instantons, Phys. Rev. D 16 (1977) 1791 [INSPIRE].

[11] S. Weinberg, A New Light Boson?, Phys. Rev. Lett. 40 (1978) 223 [InSPIRE].

[12] F. Wilczek, Problem of Strong $P$ and $T$ Invariance in the Presence of Instantons, Phys. Rev. Lett. 40 (1978) 279 [INSPIRE].

[13] V.A. Rubakov, Grand unification and heavy axion, JETP Lett. 65 (1997) 621 [hep-ph/9703409] [INSPIRE].

[14] S. Dimopoulos, A. Hook, J. Huang and G. Marques-Tavares, A collider observable QCD axion, JHEP 11 (2016) 052 [arXiv: 1606. 03097] [INSPIRE].

[15] A. Hook, Anomalous solutions to the strong CP problem, Phys. Rev. Lett. 114 (2015) 141801 [arXiv: 1411.3325] [INSPIRE].

[16] G.C. Branco, P.M. Ferreira, L. Lavoura, M.N. Rebelo, M. Sher and J.P. Silva, Theory and phenomenology of two-Higgs-doublet models, Phys. Rept. 516 (2012) 1 [arXiv:1106.0034] [INSPIRE].

[17] S.P. Martin, A Supersymmetry primer, Adv. Ser. Direct. High Energy Phys. 18 (1998) 1 [hep-ph/9709356] [INSPIRE].

[18] D.J. Miller, R. Nevzorov and P.M. Zerwas, The Higgs sector of the next-to-minimal supersymmetric standard model, Nucl. Phys. B 681 (2004) 3 [hep-ph/0304049] [InSPIRE]. 
[19] M. Bauer, M. Neubert and A. Thamm, LHC as an Axion Factory: Probing an Axion Explanation for $(g-2)_{\mu}$ with Exotic Higgs Decays, Phys. Rev. Lett. 119 (2017) 031802 [arXiv: 1704.08207] [INSPIRE].

[20] U. Ellwanger and S. Moretti, Possible Explanation of the Electron Positron Anomaly at $17 \mathrm{MeV}$ in ${ }^{8}$ Be Transitions Through a Light Pseudoscalar, JHEP 11 (2016) 039 [arXiv: 1609.01669] [INSPIRE].

[21] S. Murgia, The Fermi-LAT Galactic Center Excess: Evidence of Annihilating Dark Matter?, Ann. Rev. Nucl. Part. Sci. 70 (2020) 455 [inSPIRE].

[22] A. Berlin, D. Hooper and S.D. McDermott, Simplified Dark Matter Models for the Galactic Center Gamma-Ray Excess, Phys. Rev. D 89 (2014) 115022 [arXiv:1404.0022] [InSPIRE].

[23] ATLAS collaboration, Search for Higgs Boson Decays into a $Z$ Boson and a Light Hadronically Decaying Resonance Using $13 \mathrm{TeV}$ pp Collision Data from the ATLAS Detector, Phys. Rev. Lett. 125 (2020) 221802 [arXiv: 2004.01678] [INSPIRE].

[24] ATLAS collaboration, Search for the Higgs boson produced in association with a vector boson and decaying into two spin-zero particles in the $H \rightarrow a a \rightarrow 4 b$ channel in pp collisions at $\sqrt{s}=13 \mathrm{TeV}$ with the ATLAS detector, JHEP 10 (2018) 031 [arXiv:1806.07355] [INSPIRE].

[25] ATLAS collaboration, Search for Higgs boson decays into two new low-mass spin-0 particles in the $4 b$ channel with the ATLAS detector using pp collisions at $\sqrt{s}=13 \mathrm{TeV}$, Phys. Rev. D 102 (2020) 112006 [arXiv: 2005.12236] [INSPIRE].

[26] CMS collaboration, Search for light pseudoscalar boson pairs produced from decays of the $125 \mathrm{GeV}$ Higgs boson in final states with two muons and two nearby tracks in pp collisions at $\sqrt{s}=13 \mathrm{TeV}$, Phys. Lett. B 800 (2020) 135087 [arXiv:1907.07235] [INSPIRE].

[27] CMS collaboration, Search for a light pseudoscalar Higgs boson in the boosted $\mu \mu \tau \tau$ final state in proton-proton collisions at $\sqrt{s}=13 \mathrm{TeV}, \mathrm{JHEP} 08$ (2020) 139 [arXiv:2005.08694] [INSPIRE].

[28] ATLAS collaboration, Search for new phenomena in events with at least three photons collected in pp collisions at $\sqrt{s}=8 \mathrm{TeV}$ with the ATLAS detector, Eur. Phys. J. C 76 (2016) 210 [arXiv: 1509.05051] [inSPIRE].

[29] ATLAS collaboration, Search for Higgs boson decays into pairs of light (pseudo)scalar particles in the $\gamma \gamma j j$ final state in pp collisions at $\sqrt{s}=13 \mathrm{TeV}$ with the ATLAS detector, Phys. Lett. B 782 (2018) 750 [arXiv:1803.11145] [InSPIRE].

[30] M. Bauer, M. Neubert and A. Thamm, Collider Probes of Axion-Like Particles, JHEP 12 (2017) 044 [arXiv : 1708.00443] [INSPIRE].

[31] D. d'Enterria, Collider constraints on axion-like particles, in Workshop on Feebly Interacting Particles, (2021) [arXiv:2102.08971] [INSPIRE].

[32] B.C. Allanach, D. Bhatia and A.M. Iyer, Dissecting Multi-Photon Resonances at the Large Hadron Collider, Eur. Phys. J. C 77 (2017) 595 [arXiv: 1706.09039] [INSPIRE].

[33] A. Martin, Higgs Cascade Decays to gamma gamma + jet jet at the LHC, hep-ph/0703247 [INSPIRE].

[34] CMS collaboration, Measurements of the Higgs boson width and anomalous HVV couplings from on-shell and off-shell production in the four-lepton final state, Phys. Rev. D 99 (2019) 112003 [arXiv: 1901.00174] [INSPIRE]. 
[35] I. Brivio et al., ALPs Effective Field Theory and Collider Signatures, Eur. Phys. J. C 77 (2017) 572 [arXiv : 1701.05379] [INSPIRE].

[36] J. Jaeckel and M. Spannowsky, Probing MeV to $90 \mathrm{GeV}$ axion-like particles with LEP and LHC, Phys. Lett. B 753 (2016) 482 [arXiv:1509.00476] [inSPIRE].

[37] A. Alves, A.G. Dias and K. Sinha, Diphotons at the Z-pole in Models of the $750 \mathrm{GeV}$ Resonance Decaying to Axion-Like Particles, JHEP 08 (2016) 060 [arXiv:1606.06375] [INSPIRE].

[38] CDF collaboration, First Search for Exotic Z Boson Decays into Photons and Neutral Pions in Hadron Collisions, Phys. Rev. Lett. 112 (2014) 111803 [arXiv:1311.3282] [INSPIRE].

[39] ATLAS collaboration, Search for a Higgs boson decaying to four photons through light CP-odd scalar coupling using $4.9 \mathrm{fb}^{-1}$ of $7 \mathrm{TeV}$ pp collision data taken with ATLAS detector at the LHC, Tech. Rep. ATLAS-CONF-2012-079, CERN, Geneva (2012).

[40] J. Alwall et al., The automated computation of tree-level and next-to-leading order differential cross sections, and their matching to parton shower simulations, JHEP 07 (2014) 079 [arXiv: 1405.0301] [INSPIRE].

[41] T. Sjöstrand, S. Mrenna and P.Z. Skands, A Brief Introduction to PYTHIA 8.1, Comput. Phys. Commun. 178 (2008) 852 [arXiv:0710.3820] [INSPIRE].

[42] DELPHES 3 collaboration, DELPHES 3, A modular framework for fast simulation of a generic collider experiment, JHEP 02 (2014) 057 [arXiv: 1307.6346] [INSPIRE].

[43] ATLAS collaboration, Measurement of the cross section for inclusive isolated-photon production in pp collisions at $\sqrt{s}=13 \mathrm{TeV}$ using the ATLAS detector, Phys. Lett. B 770 (2017) 473 [arXiv: 1701.06882] [inSPIRE].

[44] S.D. Ellis, T.S. Roy and J. Scholtz, Phenomenology of Photon-Jets, Phys. Rev. D 87 (2013) 014015 [arXiv: 1210 .3657] [INSPIRE].

[45] B. Sheff, N. Steinberg and J.D. Wells, Higgs boson decays into narrow diphoton jets and their search strategies at the Large Hadron Collider, Phys. Rev. D 104 (2021) 036009 [arXiv: 2008.10568] [INSPIRE].

[46] D. Wang, L. Wu, J.M. Yang and M. Zhang, Photon-jet as a probe of axion-like particles at the $L H C$, arXiv:2102.01532 [INSPIRE].

[47] J. Ebadi, S. Khatibi and M. Mohammadi Najafabadi, New probes for axionlike particles at hadron colliders, Phys. Rev. D 100 (2019) 015016 [arXiv:1901.03061] [INSPIRE].

[48] J. Bergstra, D. Yamins and D.D. Cox, Making a science of model search: Hyperparameter optimization in hundreds of dimensions for vision architectures, proceedings of the 30th International Conference on International Conference on Machine Learning, PMLR 28 (2013) 115 [arXiv:1209.5111].

[49] A. Alves, T. Ghosh and K. Sinha, Can We Discover Double Higgs Production at the LHC?, Phys. Rev. D 96 (2017) 035022 [arXiv:1704.07395] [InSPIRE].

[50] N.V. Chawla, K.W. Bowyer, L.O. Hall and W.P. Kegelmeyer, SMOTE: Synthetic Minority Over-sampling Technique, J. Artif. Intell. Res. 16 (2002) 321 [arXiv:1106.1813] [INSPIRE].

[51] H. He, Y. Bai, E.A. Garcia and S. Li, Adasyn: Adaptive synthetic sampling approach for imbalanced learning, in 2008 IEEE International Joint Conference on Neural Networks (IEEE World Congress on Computational Intelligence), (2008), pp. 1322-1328, DOI. 
[52] A. Butter, S. Diefenbacher, G. Kasieczka, B. Nachman and T. Plehn, GANplifying event samples, SciPost Phys. 10 (2021) 139 [arXiv:2008.06545] [InSPIRE].

[53] A. Butter and T. Plehn, Generative Networks for LHC events, arXiv:2008.08558 [INSPIRE].

[54] C. Chen, O. Cerri, T.Q. Nguyen, J.-R. Vlimant and M. Pierini, Data Augmentation at the LHC through Analysis-specific Fast Simulation with Deep Learning, arXiv:2010.01835 [INSPIRE].

[55] CMS collaboration, Measurements of Higgs boson properties in the diphoton decay channel in proton-proton collisions at $\sqrt{s}=13 \mathrm{TeV}$, JHEP 11 (2018) 185 [arXiv:1804.02716] [INSPIRE]. 